\title{
On the evaluation of an Optical OFDM Radio over FSO system with IM-DD for high-speed indoor communications
}

\author{
J. Perez ${ }^{1}$, F.I. Chicharro ${ }^{1,2}$, B. Ortega ${ }^{1}$ and J. Mora ${ }^{1}$ \\ ${ }^{I}$ Photonics Research Labs (PRL), ITEAM Research Institute, Universitat Politècnica de València, Camino de \\ Vera s/n, 46022 Valencia, Spain \\ ${ }^{2}$ Institute for Multidisciplinary Mathematics IMM, Universitat Politècnica de València, Camino de Vera $s / n$, \\ 46022 Valencia, Spain
}

E-mail :joapeso@upv.es, frachilo@upv.es,.bortega@dcom.upv.es,.jmalmer@iteam.upv.es

\begin{abstract}
A novel radio over fiber (RoF) and free space optics (FSO) hybrid system based on optical OFDM (OOFDM) signal transmission is proposed in this paper to provide high capacity optical wireless indoor communication links. A low cost transmission scheme based on IM-DD is proposed to simplify and reuse existing infrastructure to deploy a high bandwidth FSO link. This paper shows the experimental results based on the implementation of the proposed scheme under a 16-QAM OOFDM digital transmission over $10 \mathrm{~km}$ Single Mode Fiber (SMF) and 300 mm FSO link. The results show the feasibility to deploy a $20 \mathrm{Gbit} / \mathrm{s}$ communication system over a $5 \mathrm{GHz} \mathrm{RF}$ bandwidth OFDM signal by using IM-DD scheme for the optical transmission without any optical multiplexing technique.
\end{abstract}

Keywords: OFDM, FSO, OWC; IM-DD, indoor, radio over fiber.

\section{INTRODUCTION}

In recent years, the increasing demand for wireless services which require high-speed, high capacity and ubiquitous connections has become essential to the development of the access networks. In this area, fiber technologies to the home (FTTH) are becoming a usual asset in residential areas to address those requirements. However, due to the implementation of $5 \mathrm{G}$ technologies and the need for ubiquitous access demand free-space optical (FSO) links as part of optical wireless communications (OWC) for mostly outdoor applications [1]-[3]. Among providing reliable high data rate optical wireless links [4] FSO links are recognized as fundamental part for the development of future $5 \mathrm{G}$ and beyond technologies [5]. The FSO technology will help to improve the capacity of such 5G wireless systems at their mobile fronthaul and backhaul [6][7].

This paper focused on the evaluation of a proposed OOFDM system for its application over a free space link to provide high-speed indoor communications. For example, dual polarization phase state modulation in combination with wavelength multiplexing techniques has been demonstrated over FSO for 16 x $100 \mathrm{Gbit} / \mathrm{s}$ high capacity optical metro and access networks [4], [8]. In this paper, we focus on the demonstration of high-speed radio over FSO system with a low cost solution based on IM-DD for indoor communications environments.

\section{SYSTEM DESCRIPTION}

The proposed experimental set-up is depicted in Fig. 1. Generation and reception of the OFDM signal is processed offline in Matlab. The transmitted signal is loaded into an Arbitrary Waveform Generator (AWG7122C, Tektronix), sampling at $24 \mathrm{GS} / \mathrm{s}$. An FFT size of 4096 with 854 16-QAM data-bearing subcarriers is used, satisfying Hermitian symmetry. The resulting OFDM signal is centered at $3.25 \mathrm{GHz}$ with a raw data rate of 20 Gbit/s. A $6.25 \%$ cyclic prefix of the symbol is applied and channel estimation and equalization areperformed in detection by the inclusion of a block-type equispaced pilot pattern of $10 \%$. A pre-emphasis filter is also applied to overcome the non-flat response of the electrical transmitter.

Table 1. System parameters

\begin{tabular}{|l|l|}
\hline OFDM BW & $3.25 \mathrm{GHz}$ \\
\hline Modulation format & $16-\mathrm{QAM}$ \\
\hline EDFA & $15 \mathrm{dBm}$ \\
\hline Laser CW wavelength & $1550 \mathrm{~nm}$ \\
\hline Optical laser power output & $8 \mathrm{dBm}$ \\
\hline MZM - Intensity modulator- BW & $10 \mathrm{GHz}$ \\
\hline MZM V VIAS & $5.3 \mathrm{~V}$ DC \\
\hline FSO link length LFSO & $300 \mathrm{~mm}$ \\
\hline
\end{tabular}

(C) 2017 IEEE. Personal use of this material is permitted. Permission from IEEE must be obtained for all other uses, in any current or future media, including reprinting/republishing this material for advertising or promotional purposes, creating new collective works, for resale.

Published at: 2017 19th International Conference on Transparent Optical Networks (ICTON) by IEEE, DOI: https://doi.org/10.1109/ICTON.2017.8025112 


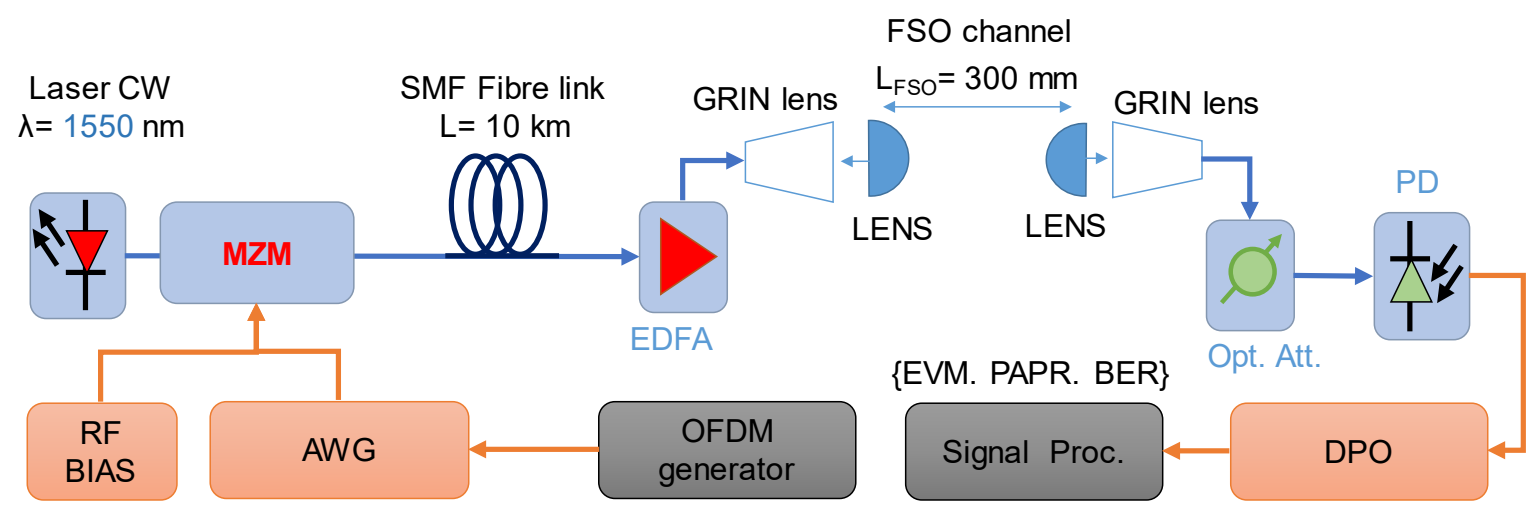

(a)

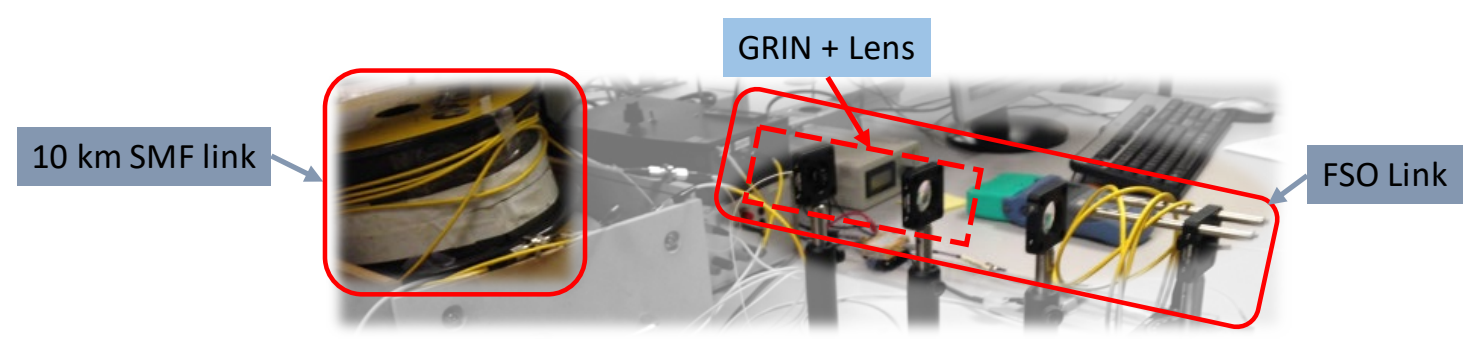

(b)

Fig. 1. System set-up (a) description and (b) snapshot of the FSO link and SMF link laboratory implementation.

Then, the RF signal is double-sideband modulated onto a Mach-Zehnder modulator fed by a CW laser source at $1550 \mathrm{~nm}$. The signal is transmitted over an optical channel formed by a SMF spool of $10 \mathrm{~km}$. The output signal from SMF is amplified by Erbium doped fiber amplifier (EDFA) to compensate loss and gain sufficient power budget. Afterwards, the optical signal launch into the FSO link - both FSO transceivers composed of graded-index lenses (GRIN) (Thorlabs 50-1550A-APC) with an aperture of $1.8 \mathrm{~mm}$ and plano-convex lenses with a diameter of $25.4 \mathrm{~mm}$. The optical signal is collimated at the receiver part and passed through an optical attenuator to emulate optical losses on the system.

The received optical signal is photodetected (U2T BPDV2020R-VF-FP) and captured electrically by a RealTime Digital Phosphor Oscilloscope (DPO72004C, Tektronix), sampled at $50 \mathrm{GS} / \mathrm{s}$, in order to process it offline. The captured samples are synchronized following a cross correlation scheme, and parallelized for processing in the regular OFDM receiver blocks. The cyclic prefix is removed before the application of the 4096-FFT. The channel estimation and equalization is performed with the information carried by the pilot symbols, and 16-QAM data are detected, recovering the transmitted bit stream after serialization. The main parameters of the overall system are summarized in Table 1 .

\section{EXPERIMENTAL RESULTS}

The electrical signal generated and received is centered at $3.25 \mathrm{GHz}$, as depicted in Fig. 2. The main results obtained are shown in Fig 3. Once the modulator is biased at the quadrature point of $5.3 \mathrm{~V}$ the signal is either detected as a back-to-back (B2B) configuration or passed through the RoF $10 \mathrm{Km}$ SMF link or over the overall hybrid system, i.e. the RoF $10 \mathrm{Km} \mathrm{SMF+FSO} \mathrm{link.} \mathrm{The} \mathrm{optical} \mathrm{attenuator} \mathrm{allows} \mathrm{evaluating} \mathrm{the} \mathrm{impact} \mathrm{of} \mathrm{the}$ attenuation losses in the system performance. The optical power received at the photoreceiver is $3 \mathrm{dBm}$ without the optical attenuator. The FSO link losses are in the range of $2 \mathrm{~dB}$ due the short range of the link and a precise process of alignment. However, it is worth to mention that the small misalignments on this experimental set-up address to accumulate losses of around $8 \mathrm{~dB}$. This evaluation procedure provided results depicted in Fig. 3 in terms of EVM and BER measured results. The FSO link range is $300 \mathrm{~mm}$ in order to emulate a high-capacity short-range optical wireless communication channel typical for indoor environments, e.g. data centers. 

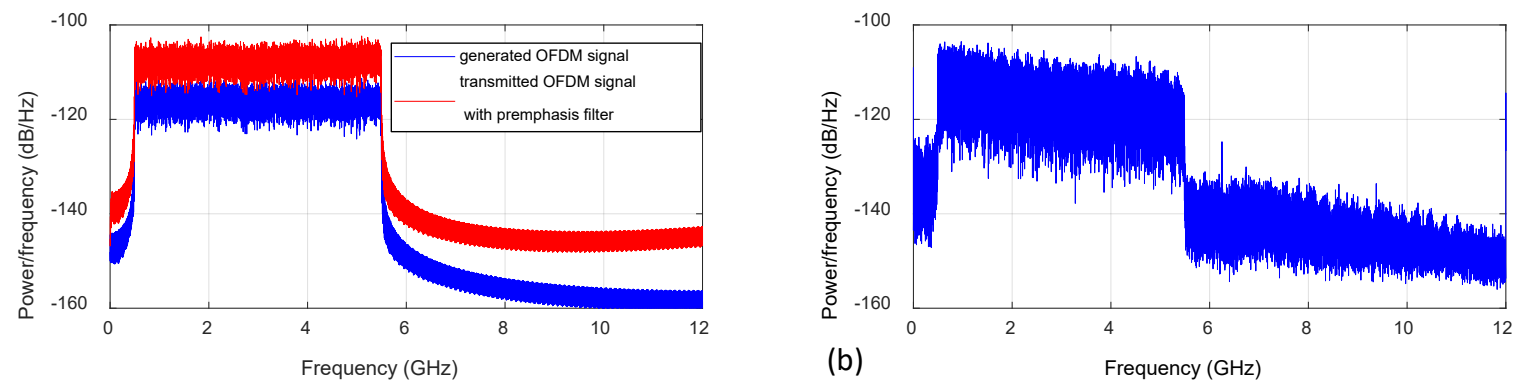

Fig. 2.(a) Generated and transmitted OFDM signal. (b) Received electrical OFDM signal.
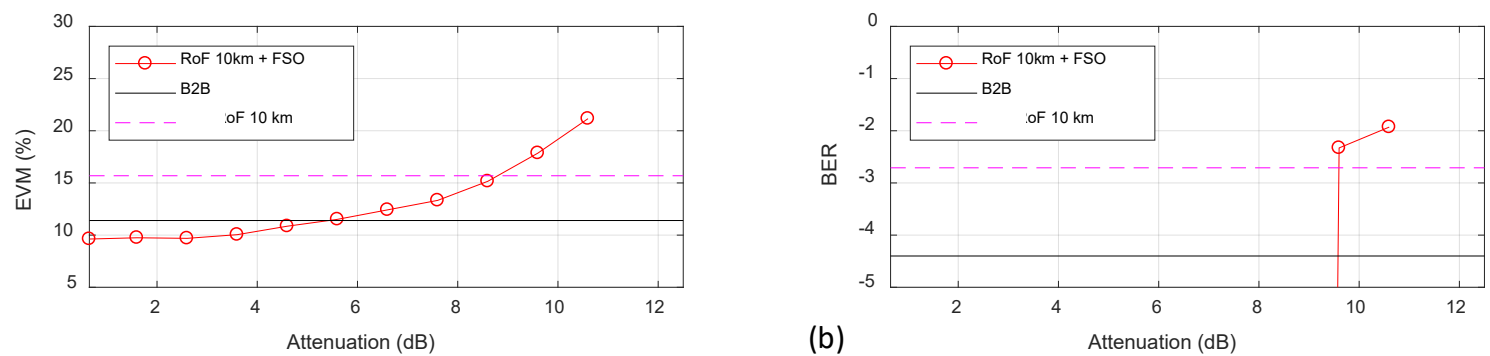

Fig. 3. Impact of the optical losses, due to optical attenuation, at the proposed 16 QAM OOFDM $10 \mathrm{~km}$ SMF radio-over-FSO system in terms of measured (a) EVM and (b) BER.

The measured EVM shown in Fig. 3(a) indicates that the inclusion of the $10 \mathrm{~km}$ SMF RoF link decreases, as expected, the quality of the system about $5 \%$ from the initial $\mathrm{B} 2 \mathrm{~B}$ configuration. However, the combination of the RoF and FSO link leads to obtaining similar values as the B2B system up to $6 \mathrm{~dB}$ of optical attenuation. Then the system starts to degrade quickly for optical attenuation values beyond 9-10 dB. On the other hand, evaluating the measured BER results, in the Fig. 3(b), shows no degradation until the aforementioned value 9-10 dB optical attenuation. The experimental EVM results indicated that no errors were detected until the optical receiver power decreases below $10 \mathrm{~dB}$ of optical attenuation,
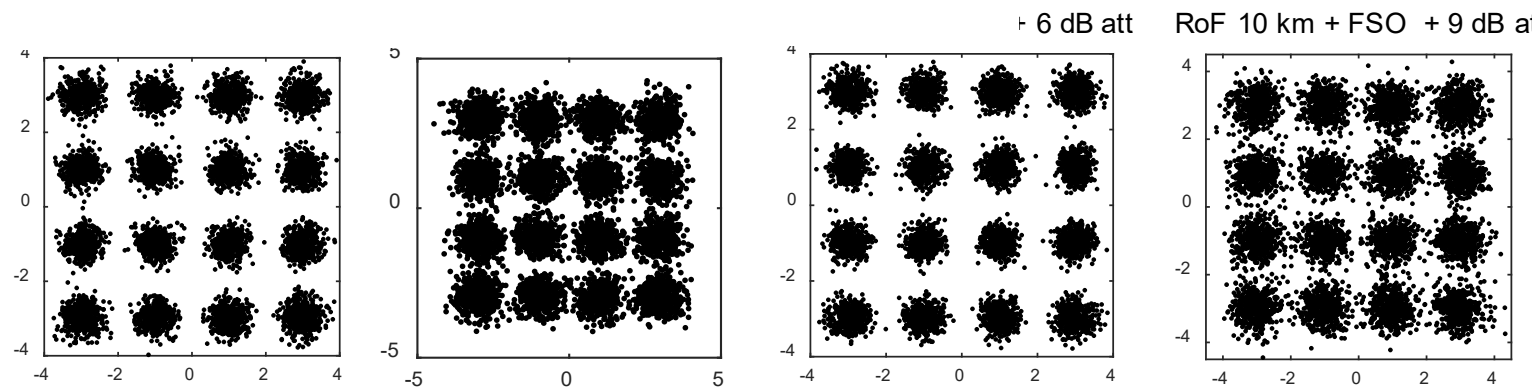

Fig. 4. 16 QAM-OOFDM signal constellations (a) B2B configuration, (b) with $10 \mathrm{Km}$ radio over fiber, (c) adding the FSO channel with optical power reduced $6 d B$ and (d) reducing the optical power $9 d B$.

The 16 QAM-OOFDM signal constellations at Fig. 4 show the corresponding degradation to different experimental parameters. It is very interesting to point out how the degradation of the performance for RoF + FSO system is comparable to the B2B and RoF system after detection and signal processing. Moreover, it is well known that the FEC limit defines the $3.8 \times 10^{-3}$ BER threshold level for a 16-QAM communication digital systems, and our proposed system accomplish such requirement when optical attenuation is less than $10 \mathrm{~dB}$ [9].

\section{CONCLUSIONS}

In this paper, we address the feasibility to build ultra-broadband high-speed radio over FSO links for short-range links indoor scenarios. The innovative introduction of IM-DD scheme allows proposing a moderate cost approach compared to previous ones which are based on balanced detection schemes, advanced modulation formats and optical multiplexing techniques. Moreover, this proposal improves the feasibility of reuse, easy deployment and 
management as one of the main characteristics of the aforementioned OWC systems as they use moderate cost optical components to deploy the final FSO link. The evaluated 16-QAM OOFDM transmission system shows the feasibility to support $20 \mathrm{Gbit} / \mathrm{s}$ over an ultra-broadband RF signal of $5 \mathrm{GHz}$ over $10 \mathrm{~km}$ fiber and $300 \mathrm{~mm}$ free space link. These results will help to pave future studies on the feasibility of high capacity optical wireless communication systems for IoTs environments.

\section{ACKNOWLEDGEMENTS}

This work was supported by the National Project funded by the Ministerio de Economía y Competitividad and the European Regional Development Fund under Grant RTC-2016-5343-7, by the Spanish MINECO Juan de la Cierva Fellowship JCI-2012-14805, and also by the Regional Project PROMETEO FASE II funded by the Generalitat Valenciana Grant II/2013/2012.

\section{REFERENCES}

[1] M. A. Khalighi and M. Uysal, "Survey on Free Space Optical Communication: A Communication Theory Perspective," IEEE Commun. Surv. Tutorials, vol. 16, no. 4, pp. 2231-2258, 2014.

[2] M. Uysal, C. Capsoni, Z. Ghassemlooy, A. Boucouvalas, and E. Udvary, Optical Wireless Communications. Cham: Springer International Publishing, 2016.

[3] E. Agrell et al., "Roadmap of optical communications," J. Opt., vol. 18, no. 6, p. 63002, Jun. 2016.

[4] G. Parca, A. Shahpari, V. Carrozzo, G. Tosi Beleffi, and A. J. Teixeira, "Optical wireless transmission at 1.6-Tbit/s $(16 \times 100 \mathrm{Gbit} / \mathrm{s})$ for next-generation convergent urban infrastructures,” Opt. Eng., vol. 52, no. 11, p. 116102, Nov. 2013.

[5] C.-X. Wang et al., "Cellular architecture and key technologies for $5 \mathrm{G}$ wireless communication networks," IEEE Commun. Mag., vol. 52, no. 2, pp. 122-130, Feb. 2014.

[6] I. Gasulla and J. Perez, "Multicore fiber opportunities for Centralized Radio Access Networks," in 2014 16th International Conference on Transparent Optical Networks (ICTON), 2014, pp. 1-4.

[7] P. Pesek, J. Bohata, S. Zvanovec, and J. Perez, "Characterization of dual-polarization analogue radio over fiber fronthaul for LTE C-RAN architecture," in 2016 10th International Symposium on Communication Systems, Networks and Digital Signal Processing (CSNDSP), 2016, pp. 1-4.

[8] A. Shahpari et al., "Ultra-High-Capacity Passive Optical Network Systems with Free-Space Optical Communications," Fiber Integr. Opt., vol. 33, no. 3, pp. 149-162, May 2014.

[9] A. Alvarado, E. Agrell, D. Lavery, R. Maher, and P. Bayvel, "Replacing the Soft-Decision FEC Limit Paradigm in the Design of Optical Communication Systems," J. Light. Technol., vol. 33, no. 20, pp. 43384352, Oct. 2015. 